\section{Highlights of the San Antonio Breast Cancer Symposium 2015: part 2}

\author{
John R Benson ${ }^{*, 1} \&$ Ismail Jatoi²
}

The 38th annual San Antonio Breast Cancer Symposium was convened in San Antonio (TX, USA) on 8-12 December 2015. More than 7000 clinicians and scientists from around the world participated in the symposium, which featured a range of presentations and keynote talks pertaining to breast cancer screening, prevention, loco-regional and systemic therapies. This two-part report highlights a selection of important studies presented at this premier breast cancer event with part 1 focusing on radiotherapy treatment, translational approaches to immunotherapy and longer term cardiotoxicity from anti-HER2 therapies. The second part of this report will discuss a range of topics including de-escalation of chemotherapy regimens in luminal A tumors, the prognostic relevance of circulating tumor cells, the optimum treatment of triple-negative breast cancer and shifting attitudes to primary surgical treatment.

First draft submitted: 22 January 2016; Accepted for publication: 1 February 2016; Published online: 15 February 2016

Much research effort is currently directed toward identifying patients in whom chemotherapy can be safely omitted. Intrinsic tumor subtypes have proven prognostic benefit and are increasingly being incorporated into the clinical decision-making process. Torsten Neilsen (University of British Columbia, Vancouver, Canada) presented results of a formal prospective-retrospective study to assess the hypothesis that patients with luminal A tumors derive minimal benefit from chemotherapy. Heterogeneity tests for interaction were applied to historical Phase III randomized trials comparing chemotherapy with no chemotherapy. More than 1000 premenopausal, node-positive women with tumors greater than $5 \mathrm{~cm}$ and unknown hormone receptor and HER2 status undergoing mastectomy, axillary dissection and radiotherapy were available for analysis. Trial arms included control, levamisole, cyclophosphamide (C) and cyclophosphamide, methotrexate and 5-fluorouracil (CMF). Tumor subtyping was determined by immunohistochemistry combined with tissue microarrays and interpreted by pathologists with no access to patient data. Luminal A tumors were defined asestrogen receptor-positive, progesterone receptor $>20 \%$, Ki- $67 \leq 13 \%$ and HER2 negative [1]. The chemotherapy regimens $\mathrm{C}$ and CMF were of comparable efficacy and improved both 10-year disease-free and 25-year overall survival. Notwithstanding problems of retrospective analyses and limited analytic validity for $\mathrm{Ki}-67$, there was no significant benefit from chemotherapy (hazard ratio $[\mathrm{HR}]$ : 1.07; test for interaction $\mathrm{p}<0.05)$ for those tumors classified as luminal $\mathrm{A}(\mathrm{n}=165)$ in contrast to nonluminal A tumors $(n=468)$ with evident benefit from chemotherapy (HR: 0.5$)$.

Detection of circulating tumors cells (CTCs) is a promising method for early treatment monitoring in metastatic breast cancer and potentially as a surveillance marker for patients with minimal

'Cambridge Breast Unit, Addenbrookes Hospital \& University of Cambridge, Cambridge, UK

2Division of Surgical Oncology, Dale H Dorn Chair in Surgery, University of Texas Health Science Centre, San Antonio, TX, USA

*Author for correspondence: Tel.: +44 1223 586577; Fax: +44 1223 586932; john.benson@addenbrookes.nhs.uk

\section{KEYWORDS}

- breast-conserving surgery

- mastectomy $\bullet$ radiation

treatment $\bullet$ San Antonio

- triple-negative cancer 
residual disease but high risk of relapse. The prognostic relevance of CTCs has been established for both primary and metastatic breast cancer [2] but data are lacking on their clinical utility during longer term follow-up of breast cancer patients. Wolfgang Janni (Universitatsklinikum Ulm, Germany) examined the prevalence of CTCs among a group of 1087 patients 2 years after administration of adjuvant chemotherapy. Immunomagnetic enrichment with an epithelial cell adhesion molecule antibody and labeling of cells with an anti-cytokeratin antibody was used to detect CTCs; a median of one CTC was found in the bloodstream of 198 patients (18.2\%) 2 years after chemotherapy treatment (range 1-99) and was a significant independent prognostic factor for poorer overall (HR: 3.82; 95\% CI: 1.99-7.39) and disease-free survival (HR: 2.28; 95\% CI: 1.48-3.50) with a p-value of $<0.001$. Multivariate analysis confirmed the prognostic impact of CTCs after adjustment for age, menopausal status, tumor size, nodal, hormonal and HER2 status in addition to presence of CTCs before chemotherapy. Patients with CTCs both before and after chemotherapy had a particularly poor outcome and there was no relationship between the presence of CTCs and biological subtype.

Recent studies on bisphosphonates have been practice changing and led to incorporation of these agents into adjuvant systemic schedules for postmenopausal women with early-stage hormone receptor-positive disease for whom there is evidence on meta-analysis of improved disease-free and overall survival [3]. Michael Gnant (Medical University of Vienna, Austria) provided further data from the ABCSG-18 (Austrian Breast and Colorectal Cancer Study Group) trial on use of the human monoclonal antibody (IgG2) denosumab in 3425 postmenopausal breast cancer patients. In a randomized double-blind trial, denosumab was found to yield an $18 \%$ improvement in disease-free survival similar to the published meta-analysis on bisphosphonates [3]. Denosumab is not associated with an increase in adverse side effects but significantly reduces the incidence of clinical and vertebral fractures and enhances bone mineral density. Gnant believes this agent should be offered to postmenopausal women receiving aromatase inhibitor therapy.

With an inspirational presentation, Ratna Vadlamudi (University of Texas Health Science Center, TX, USA) discussed strategies based on disrupting ESR1 signaling by targeting the interaction between ESR1 and its co-regulators. His group has developed a specific ESR1-binding inhibitor (ECBI) which mimics the co-regulator nuclear receptor box motif and competes for binding to ESR1. This in turns prevents formation of an active receptor/co-activator complex and has been shown in cell proliferation assays to be an effective approach against certain breast cancer cells (e.g. MCF-7) which are resistant to hormonal manipulation with either tamoxifen or an aromatase inhibitor (letrozole). Interestingly, ECBIs do not affect growth of triple-negative breast cancer (TNBC) cells and are active in a dose-dependent manner with oral bioavailability and minimal toxicity in mouse models.

The risk of relapse after trastuzumab-based adjuvant therapy is highest in the first 12 months after treatment [4]. Arlene Chan (Breast Cancer Research Centre at Western Australia and Curtin University, Australia) presented results of a Phase III randomized trial assessing the value of an irreversible pan-HER tyrosine kinase inhibitor (neratinib) following standard anti-HER2 adjuvant therapy. The benefits of neratinib in terms of disease-free survival were greatest for estrogen receptor-positive patients with centrally confirmed HER-positive status enrolled within 12 months of completing trastuzumab treatment. This was an exploratory analysis and results cannot be extrapolated to patients relapsing after pertuzumab treatment.

Marissa van Maaren from The Netherlands Cancer Institute delivered somewhat intriguing findings from an observational study comparing breast-conserving surgery (BCS) with mastectomy in terms of disease-free and overall survival at 10 years. The primary cohort included 37,207 patients treated between 2000 and 2004 analyzed for 10 -year overall survival and a second cohort of 7552 patients treated from 2003 assessed for distant metastases-free survival at 10 years. All patients had early-stage disease (T1-2N0-1) and 58.4\% underwent BCS followed by breast radiotherapy - this group of women tended to be younger with smaller, welldifferentiated tumors. At a median follow-up of 11.3 years, there was a statistically significant overall survival advantage for BCS compared with mastectomy irrespective of tumor stage (T1N0 and T1N1) with a HR of 0.81 (95\% CI: $0.78-0.85 ; \mathrm{p}<0.001)$. For the 2003 subgroup, there was no difference in distant metastasisfree survival at 10 years for patients with T1N1 
tumors but a significant benefit for BCS in the T1N0 subgroup $(\mathrm{p}=0.014)$. As discussant for this paper, one of the authors (I Jatoi) considered interpretation to be severely limited by selection bias and unmeasured confounding factors. The latter have invalidated studies on outcomes of contralateral prophylactic mastectomy (CPM) in which observed reductions of nonbreast cancer deaths cannot be attributed to any surgical intervention [5]. Thus BCS when compared with mastectomy and bilateral mastectomy for unilateral cancer when compared with unilateral mastectomy or BCS have been associated with improved survival. In both instances, the better survival of one group of patients versus another in these observational studies is likely due to unmeasured confounders. Nonetheless, it was re-iterated that BCS remains the optimal surgical choice for most patients diagnosed with early-stage breast cancer nowadays. Women do have choices and perhaps ironically there is a trend toward maximal surgery with bilateral mastectomy and reconstruction for small unilateral cancers in women without a strong family history of breast cancer. This trend toward increased utilization of bilateral mastectomy for unilateral breast cancer is particularly pronounced in the USA but also evident in the UK and other countries. In the William L MacGuire Memorial Lecture, Norman Wolmark (Allegheny General Hospital, PA, USA) alluded to the powerful influence of social networking internet sites - these may have generated an element of distrust for doctors and science among breast cancer patients who fear recurrence and wish to minimize this risk. In essence, for younger women ( $<40$ years), the surgical option of BCS with RT has been replaced with unilateral mastectomy and CPM leading to polarization of surgical treatment.

In the 50th anniversary year since the discovery of platinum salts by Barnett Rosenberg (1924-2009), there continues to be much discussion about use of these agents for treatment of TNBC. In the discussant session, Angela DeMichele (University of Pennsylvania, PA, USA) asked whether there was sufficient evidence from clinical trials to warrant routine use of carboplatin in early-stage TNBC and whether a pathological complete response is a valid surrogate for survival outcomes? She questioned the consistency of results and their statistical rigor and ultimate clinical relevance. There is additional toxicity from carboplatin and at present there is no specific biomarker to reliably select patients for routine treatment. Gunter von Minckwitz (German Breast Group, Neu-Isenburg, Germany) presented further results of the GEPARSIXTO trial evaluating the addition of carboplatin to anthracycline (doxorubicin)/taxane (paclitaxel) based neoadjuvant chemotherapy. Carboplatin increased pCR by $17.4 \%$ from 36.9 to $53.2 \%$ ( $p=0.005)$ in TNBC patients only. Although germline BRCA status was a significant predictor of pCR, results were inconclusive for effects of carboplatin within this subgroup [6]. Diseasefree survival data at a median follow-up of 35 months were reported at SABCS 2015 with an overall benefit for carboplatin (HR: 0.81; 95\% CI: $0.54-1.21 ; \mathrm{p}=0.3115)$ and a highly favorable effect in TNBC (HR: 0.56; 95\% CI: 0.33-0.96; $p=0.035)$. DeMichelle commented that the German trial showed improved overall prognosis and a larger incremental effect of carboplatin than the CALGB40603 trial. She surmized that this might be related to a more intense chemotherapy backbone with concurrent administration of anthracycline and taxane thereby permitting a degree of 'pharmacological synergy'. It was concluded that use of platinum for early-stage TNBC should remain an individualized decision at this time of clinical equipoise and cannot be recommended for inclusion in routine treatment schedules. Well-designed trials are required which are adequately powered to detect improvements in event-free survival end points and not just $\mathrm{pCR}$, although the latter is a strong prognostic parameter in patient-level analysis [7].

The SABCS 2015 featured work of many investigators not mentioned above but who have worked diligently to reduce to the burden of breast cancer. More importantly, the symposium continues to demonstrate the courage of thousands of women around the world who have participated in clinical trials and contributed to advances in the field of both clinical and translation research. Chuck Perou (University of North Carolina, NC, USA) conceded at this meeting that the development of personalized medicine is proving to be more challenging than anticipated and precision medicine is probably a more realistic goal. Future progress may necessitate 'academic industrial collaboration' in order to perform efficient candidate gene analysis which can identify mutations which are biologically plausible, actionable and of clinical consequence. 
Financial \& competing interests disclosure

JR Benson was on the Planning Committee and I Jatoi on the Executive Committee for the 2015 San Antonio Breast Cancer Symposium. The authors have no other relevant affiliations or financial involvement with any organization or entity with a financial interest in or financial conflict with the subject matter or materials discussed in the manuscript apart from those disclosed.

No writing assistance was utilized in the production of this manuscript.

\section{References}

1 Prat A, Cheang MC, Martín M et al. Prognostic significance of progesterone receptor-positive tumor cells within immunohistochemically defined luminal A breast cancer. J. Clin. Oncol. 31(2), 203-209 (2013).

2 Rack B, Schindlbeck C, Juckstock J et al. Circulating tumor cells predict survival in early average-to-high risk breast cancer patients. J. Natl Cancer Inst. 106(5), dju066 (2014).
3 Early Breast Cancer Trialists' Collaborative Group (EBCTCG). Adjuvant bisphosphonate treatment in early breast cancer: meta-analyses of individual patient data from randomized trials. Lancet 386(10001), 1353-1361 (2015).

4 Gallagher CM, More K, Masaquel A et al. Relapse impact on overall survival in trastuzumab-treated women with HER positive early stage breast cancer. J. Clin. Oncol. 32(Suppl.) Abstract el1601 (2014).

5 Jatoi I, Parsons HM. Contralateral prophylactic mastectomy and its association with reduced mortality: evidence for selection bias. Breast Cancer Res. Treat. 148(2), 389-396 (2014).

6 von Minckwitz G, Schneeweiss A, Loibl S et al. Neoadjuvant carboplatin in patients with triple-negative and HER2-positive early breast cancer (GeparSixto; GBG 66): a randomized Phase 2 trial. Lancet Oncol. 15(7), 747-756 (2014).

7 Cortazar P, Zhang L, Untch M et al. Pathological complete response and long-term clinical benefit in breast cancer: the CTNeoBC pooled analysis. Lancet 384(9938), 164-172 (2014). 